\title{
Hyers-Ulam Stability for a Class of Quadratic Functional Equations via a Typical Form
}

\author{
Chang Il Kim, ${ }^{1}$ Giljun Han, ${ }^{1}$ and Seong-A. Shim ${ }^{2}$ \\ ${ }^{1}$ Department of Mathematics Education, Dankook University, 152 Jukjeon-ro, Suji-gu, Yongin-si, \\ Gyeonggi-do 448-701, Republic of Korea \\ ${ }^{2}$ Department of Mathematics, Sungshin Women's University, 249-1 Dongseon-dong 3-ga, Seongbuk-gu, \\ Seoul 136-742, Republic of Korea
}

Correspondence should be addressed to Giljun Han; gilhan@dankook.ac.kr

Received 29 July 2013; Accepted 7 October 2013

Academic Editor: Elena Braverman

Copyright (C) 2013 Chang Il Kim et al. This is an open access article distributed under the Creative Commons Attribution License, which permits unrestricted use, distribution, and reproduction in any medium, provided the original work is properly cited.

We investigate the following typical form of a certain class of quadratic functional equations: $f(a x+b y)+f(a x-b y)+c[f(x+$ $y)+f(x-y)-2 f(x)-2 f(y)]=2 a^{2} f(x)+2 b^{2} f(y)$. Furthermore, we provide a systematic program to prove the generalized Hyers-Ulam stability for the class of functional equations via the stability for the typical form.

\section{Introduction}

In 1964, Ulam [1] proposed the following stability problem:

"let $G_{1}$ be a group and $G_{2}$ a metric group with the metric $d$. Given a constant $\delta>0$, does there exist a constant $c>0$ such that if a mapping $f: G_{1} \rightarrow$ $G_{2}$ satisfies $d(f(x y), f(x) f(y))<c$ for all $x, y \in$ $G_{1}$, then there exists a unique homomorphism $h$ : $G_{1} \rightarrow G_{2}$ withd $(f(x), h(x))<\delta$ for all $x \in G_{1}$ ?"

In 1941, Hyers [2] answered this problem under the assumption that the groups are Banach spaces. Aoki [3] and Rassias [4] generalized the result of Hyers. Rassias [4] solved the generalized Hyers-Ulam stability of the functional inequality

$$
\|f(x+y)-f(x)-f(y)\| \leq \epsilon\left(\|x\|^{p}+\|y\|^{p}\right)
$$

for some $\epsilon \geq 0$ and $p$ with $p<1$ and for all $x, y \in X$, where $f: X \rightarrow Y$ is a function between Banach spaces. The paper of Rassias [4] has provided a lot of influence in the development of what we call the generalized Hyers-Ulam stability or the Hyers-Ulam-Rassias stability of functional equations. A generalization of the Rassias theorem was obtained by Găvruța [5] by replacing the unbounded Cauchy difference by a general control function in the spirit of Rassias approach.

The functional equation

$$
f(x+y)+f(x-y)=2 f(x)+2 f(y)
$$

is called a quadratic functional equation and a solution of a quadratic functional equation is called quadratic. A generalized Hyers-Ulam stability problem for the quadratic functional equation was proved by Skof [6] for mappings $f: X \rightarrow Y$, where $X$ is a normed space and $Y$ is a Banach space. Cholewa [7] noticed that the theorem of Skof is still true if the relevant domain $X$ is replaced by an Abelian group. Czerwik [8] proved the generalized Hyers-Ulam stability for the quadratic functional equation, and Park [9] proved the generalized Hyers-Ulam stability of the quadratic functional equation in Banach modules over a $C^{*}$-algebra. Also, the stability problems of functional equations related to quadratic functions can be found in many papers (e.g., [10, 11], etc.).

Rassias [12] investigated the following Euler-Lagrange functional equation:

$$
f(a x+b y)+f(b x-a y)=2\left(a^{2}+b^{2}\right)[f(x)+f(y)],
$$


and Gordji and Khodaei [13] investigated other EulerLagrange functional equations

$$
\begin{aligned}
f(a x+b y)+f(a x-b y) & \\
= & \frac{b(a+b)}{2} f(x+y)+\frac{b(a+b)}{2} f(x-y) \\
& +\left(2 a^{2}-a b-b^{2}\right) f(x)+\left(b^{2}-a b\right) f(y)
\end{aligned}
$$

for fixed integers $a, b$ with $b \neq a,-a,-3 a$, and

$$
f(a x+b y)+f(a x-b y)=2 a^{2} f(x)+2 b^{2} f(y)
$$

for fixed integers $a, b$ with $a^{2} \neq b^{2}$ and $a b \neq 0$.

In this paper, we consider the sum of two functional equations (2) and (5), that is,

$$
\begin{aligned}
& f(a x+b y)+f(a x-b y) \\
& +c[f(x+y)+f(x-y)-2 f(x)-2 f(y)] \\
& =2 a^{2} f(x)+2 b^{2} f(y)
\end{aligned}
$$

for fixed nonzero real numbers $a, b, c$ with $a \neq 1,-1$ and $a^{2} \neq b^{2}$, and prove the generalized Hyers-Ulam stability for it.

As applications of theorems in Sections 2 and 3, we have a systematic program to prove the generalized Hyers-Ulam stability for functional inequalities which can be deformed into the following functional inequality:

$$
\begin{aligned}
& \| f(a x+b y)+f(a x-b y)-2 a^{2} f(x)-2 b^{2} f(y) \\
& \quad+c[f(x+y)+f(x-y)-2 f(x)-2 f(y)] \| \leq \phi(x, y) .
\end{aligned}
$$

Throughout this paper, assume that $X$ is a normed space and $Y$ is a Banach space.

\section{Solutions of (6)}

In this section, we investigate solutions of (6). In Corollary 5 , it can be concluded that any solution of (6) is quadratic if $a$ is a rational number. We start with the following lemma.

Lemma 1. Let $f: X \rightarrow Y$ be a mapping with $f(0)=0$. Suppose that $f$ satisfies (6); then the following equation holds:

$$
\begin{aligned}
\left(c+\frac{c^{2}}{a^{2}}\right)[f(2 x+y)+f(2 x-y)] \\
\quad-2\left(b^{2}+2 c+\frac{c^{2}}{a^{2}}\right)[f(x+y)+f(x-y)] \\
\quad+\left(4 b^{2}+5 c+\frac{b^{2} c}{a^{2}}+\frac{2 c^{2}}{a^{2}}\right) f(y) \\
+\left(c-\frac{b^{2} c}{a^{2}}\right) f(-y)+4\left(b^{2}+2 c+\frac{c^{2}}{a^{2}}\right) f(x) \\
\quad-2\left(c+\frac{c^{2}}{a^{2}}\right) f(2 x)=0
\end{aligned}
$$

Proof. Letting $y=0$ in (6), we have

$$
f(a x)=a^{2} f(x)
$$

for all $x \in X$. Setting $x=0$ and $y=x$ in (6), we have

$$
f(b x)+f(-b x)+c[f(-x)-f(x)]=2 b^{2} f(x)
$$

for all $x \in X$. Letting $x=-x$ in (10) and adding the two equations, we have

$$
f(b x)+f(-b x)=b^{2}[f(x)+f(-x)]
$$

for all $x \in X$.

Replacing $y$ by $x+y$ in (6), we have

$$
\begin{aligned}
& f(a x+b(x+y))+f(a x-b(x+y)) \\
& \quad+c[f(2 x+y)+f(-y)] \\
& =2\left(a^{2}+c\right) f(x)+2\left(b^{2}+c\right) f(x+y)
\end{aligned}
$$

for all $x, y \in X$, and letting $y=-y$ in (12), we have

$$
\begin{aligned}
& f(a x+b(x-y))+f(a x-b(x-y)) \\
& \quad+c[f(2 x-y)+f(y)] \\
& =2\left(a^{2}+c\right) f(x)+2\left(b^{2}+c\right) f(x-y)
\end{aligned}
$$

for all $x, y \in X$.

Replacing $x$ and $y$ by $x+(b / a) y$ and $x$ in (6), respectively, we have

$$
\begin{aligned}
& f(a x+b(x+y))+f(a x-b(x-y)) \\
& \quad+c\left[f\left(2 x+\frac{b}{a} y\right)+f\left(\frac{b}{a} y\right)\right] \\
& =2\left(a^{2}+c\right) f\left(x+\frac{b}{a} y\right)+2\left(b^{2}+c\right) f(x)
\end{aligned}
$$

for all $x, y \in X$, and letting $y=-y$ in (14), we have

$$
\begin{aligned}
& f(a x+b(x-y))+f(a x-b(x+y)) \\
& \quad+c\left[f\left(2 x-\frac{b}{a} y\right)+f\left(-\frac{b}{a} y\right)\right] \\
& =2\left(a^{2}+c\right) f\left(x-\frac{b}{a} y\right)+2\left(b^{2}+c\right) f(x)
\end{aligned}
$$

for all $x, y \in X$. By (12), (13), (14), and (15), we have

$$
\begin{aligned}
c[f & (2 x+y)+f(2 x-y)+f(y)+f(-y)] \\
& -\frac{b^{2} c}{a^{2}}[f(y)+f(-y)] \\
& -\frac{c}{a^{2}}[f(2 a x+b y)+f(2 a x-b y)] \\
= & 4\left(a^{2}-b^{2}\right) f(x)+2\left(b^{2}+c\right)[f(x+y)+f(x-y)] \\
& -2\left(1+\frac{c}{a^{2}}\right)[f(a x+b y)+f(a x-b y)]
\end{aligned}
$$

for all $x, y \in X$. 
for all $x, y \in X$. By (6) and (16), we have

$$
\begin{aligned}
& c[f(2 x+y)+f(2 x-y)+f(y)+f(-y)] \\
& \quad-\frac{b^{2} c}{a^{2}}[f(y)+f(-y)] \\
& +\frac{c^{2}}{a^{2}}[f(2 x+y)+f(2 x-y)-2 f(2 x)-2 f(y) \\
& =4\left(a^{2}-b^{2}\right) f(x)+2\left(b^{2}+c\right)[f(x+y)+f(x-y)] \\
& +2 c\left(1+\frac{c}{a^{2}}\right)[f(x+y)+f(x-y)-2 f(x) \\
& \left.\quad-2 f(y)-2 a^{2} f(x)-2 b^{2} f(y)\right]
\end{aligned}
$$

for all $x, y \in X$. Now, just simplifying this equation, we can get the result.

Next three theorems deal with (6) for the different cases.

Theorem 2. Let $f: X \rightarrow Y$ be a mapping with $f(0)=0$. Suppose that $f$ satisfies (6). If $c \neq-b^{2}$ and $c^{2} \neq a^{2} b^{2}$, then $f$ is quadratic.

Proof. By (10) and (11) in the proof of Lemma 1, we have

$$
\left(b^{2}+c\right) f(x)=\left(b^{2}+c\right) f(-x)
$$

for all $x \in X$. Since $b^{2}+c \neq 0$, we have

$$
f(-x)=f(x)
$$

for all $x \in X$. By (10) and (19), we have

$$
f(b x)=b^{2} f(x)
$$

for all $x \in X$. Replacing $x$ and $y$ by $b x$ and $a y$ in (6), respectively, by (20), we have

$$
\begin{aligned}
a^{2} b^{2} & {[f(x+y)+f(x-y)] } \\
& +c\left[f(b x+a y)+f(b x-a y)-2 b^{2} f(x)-2 a^{2} f(y)\right] \\
= & 2 a^{2} b^{2}[f(x)+f(y)]
\end{aligned}
$$

for all $x, y \in X$. Replacing $x$ and $y$ by $y$ and $x$ in (6), respectively, by (19), we have

$$
\begin{aligned}
f(b x & +a y)+f(b x-a y) \\
& +c[f(x+y)+f(x-y)-2 f(x)-2 f(y)] \\
= & 2 a^{2} f(y)+2 b^{2} f(x)
\end{aligned}
$$

for all $x, y \in X$. By (21) and (22), we have

$$
\left(a^{2} b^{2}-c^{2}\right)[f(x-y)+f(x+y)-2 f(x)-2 f(y)]=0
$$

for all $x, y \in X$. Since $a^{2} b^{2}-c^{2} \neq 0$, then

$$
f(x+y)+f(x-y)=2 f(x)+2 f(y)
$$

for all $x, y \in X$. Hence, $f$ is quadratic.

Theorem 3. Let $f: X \rightarrow Y$ be a mapping with $f(0)=0$. Suppose that $f$ satisfies (6) and $a$ is a rational number. If $c^{2}=$ $a^{2} b^{2}$, then $f$ is quadratic.

Proof. Since $b^{2}+c=b(b \pm a) \neq 0$, by the first few lines in the proof of Theorem $2, f$ is even. Hence, in this case, we can easily check that (8) can be reduced to

$$
\begin{gathered}
b(b \pm a)[f(2 x+y)+f(2 x-y)] \\
=b(b \pm a)[4 f(x+y)+4 f(x-y)-6 f(y) \\
+8 f(x)-2 f(2 x)]
\end{gathered}
$$

for all $x, y \in X$. Since $b(b \pm a) \neq 0$, we have

$$
\begin{gathered}
f(2 x+y)+f(2 x-y)=4 f(x+y)+4 f(x-y)-6 f(y) \\
+8 f(x)-2 f(2 x)
\end{gathered}
$$

for all $x, y \in X$. By [14], a function $f$ satisfying (26) is quarticquadratic. But in our case, $f$ also satisfies (26) and since $f(a x)=a^{2} f(x), f$ is quadratic.

Theorem 4. Let $f: X \rightarrow Y$ be a mapping with $f(0)=0$. Suppose that $f$ satisfies (6) and $a$ is a rational number. If $c=$ $-b^{2}$, then $f$ is quadratic.

Proof. Suppose that $c=-b^{2}$. By (8), we have

$$
\begin{aligned}
& \frac{b^{2}}{a^{2}}\left(b^{2}-a^{2}\right)[f(2 x+y)+f(2 x-y)] \\
&=\frac{b^{2}}{a^{2}}\left(b^{2}-a^{2}\right)[2 f(x+y)+2 f(x-y)-4 f(x) \\
&+2 f(2 x)-f(y)-f(-y)]
\end{aligned}
$$

for all $x, y \in X$. By [15], $f$ is quadratic-cubic and since $f(a x)=a^{2} f(x), f$ is quadratic.

Combining Theorems 2, 3, and 4 we can get the following corollary as the conclusion of this section.

Corollary 5. Let $f: X \rightarrow Y$ be a mapping with $f(0)=0$. Suppose that $f$ satisfies (6) and $a$ is a rational number. Then $f$ is quadratic. 


\section{The Generalized Hyers-Ulam Stability for (6)}

In this section, we will prove the generalized Hyers-Ulam stability for (6).

Theorem 6. Let $\phi: X^{2} \rightarrow[0, \infty)$ be a function such that

$$
\sum_{n=0}^{\infty} a^{-2 n} \phi\left(a^{n} x, a^{n} y\right)<\infty
$$

for all $x, y \in X$. Let $f: X \rightarrow Y$ be a mapping such that

$$
\begin{aligned}
& \| f(a x+b y)+f(a x-b y)-2 a^{2} f(x)-2 b^{2} f(y) \\
& +c[f(x+y)+f(x-y)-2 f(x)-2 f(y)] \| \\
& \quad \leq \phi(x, y)
\end{aligned}
$$

for a fixed rational number $a$ and fixed nonzero real numbers $b, c$ with $a \neq 1,0-1$ and $a^{2} \neq b^{2}$. Then there exists a unique quadratic mapping $Q: X \rightarrow Y$ satisfying (6) and

$$
\begin{aligned}
& \|Q(x)-f(x)-f(0)\| \\
& \quad \leq \frac{1}{2} \sum_{n=0}^{\infty} a^{-2(n+1)}\left[\phi\left(a^{n} x, 0\right)+\phi(0,0)\right]
\end{aligned}
$$

for all $x \in X$.

Proof. Let $g(x)=f(x)-f(0)$. Then $g(0)=0$ and

$$
\begin{aligned}
& \| g(a x+b y)+g(a x-b y)-2 a^{2} g(x)-2 b^{2} g(y) \\
& +c[g(x+y)+g(x-y)-2 g(x)-2 g(y)] \| \\
& \quad \leq \phi(x, y)+\phi(0,0)
\end{aligned}
$$

for all $x, y \in X$. Setting $y=0$ in (31), we have

$$
\left\|a^{2} g(x)-g(a x)\right\| \leq \frac{1}{2} \phi_{0}(x, 0)
$$

for all $x \in X$, where $\phi_{0}(x, y)=\phi(x, y)+\phi(0,0)$. Replacing $x$ by $a^{n} x$ in (32) and dividing (32) by $a^{2(n+1)}$, we have

$$
\left\|a^{-2 n} g\left(a^{n} x\right)-a^{-2(n+1)} g\left(a^{n+1} x\right)\right\| \leq \frac{1}{2} a^{-2(n+1)} \phi_{0}\left(a^{n} x, 0\right)
$$

for all $x \in X$ and all nonnegative integers $n$. For $m, n \in \mathbb{N} \cup\{0\}$ with $m<n$,

$$
\begin{aligned}
\left\|a^{-2 m} g\left(a^{m} x\right)-a^{-2 n} g\left(a^{n} x\right)\right\| & \leq \frac{1}{2} \sum_{k=m}^{n-1} a^{-2(k+1)} \phi_{0}\left(a^{k} x, 0\right) \\
& =\frac{1}{2 a^{2}} \sum_{k=m}^{n-1} a^{-2 k} \phi_{0}\left(a^{k} x, 0\right)
\end{aligned}
$$

for all $x \in X$. Since (28) holds for $y=0, \sum_{k=m}^{n-1} a^{-2 k} \phi_{0}\left(a^{k} x, 0\right)$ goes to 0 as $m \rightarrow \infty$. So $\left\{a^{-2 n} g\left(a^{n} x\right)\right\}$ is a Cauchy sequence in $Y$, and since $Y$ is a Banach space, there exists a mapping $Q: X \rightarrow Y$ such that

$$
Q(x)=\lim _{n \rightarrow \infty} a^{-2 n} g\left(a^{n} x\right)
$$

for all $x \in X$ and

$$
\|Q(x)-g(x)\| \leq \frac{1}{2} \sum_{n=0}^{\infty} a^{-2(n+1)} \phi_{0}\left(a^{n} x, 0\right)
$$

for all $x \in X$. Replacing $x$ and $y$ by $a^{n} x$ and $a^{n} y$ in (31), respectively, and dividing (31) by $a^{2 n}$, we have

$$
\begin{aligned}
& \| a^{-2 n} g\left(a^{n}(a x+b y)\right)+a^{-2 n} g\left(a^{n}(a x-b y)\right) \\
& -c\left[a^{-2 n} g\left(a^{n}(x+y)\right)+a^{-2 n} g\left(a^{n}(x-y)\right)\right. \\
& \left.-2 \cdot a^{-2 n} g\left(a^{n} x\right)-2 \cdot a^{-2 n} g\left(a^{n} y\right)\right] \\
& -2 \cdot a^{2} \cdot a^{-2 n} g\left(a^{n} x\right)-2 \cdot b^{2} \cdot a^{-2 n} g\left(a^{n} y\right) \| \\
& \leq a^{-2 n} \phi_{0}\left(a^{n} x, a^{n} y\right)
\end{aligned}
$$

for all $x, y \in X$ and letting $n \rightarrow \infty$ in the above inequality, we can show that $Q$ satisfies (6). By Corollary 5, $Q$ is quadratic.

Now, we show the uniqueness of the quadratic mapping $Q$. Suppose that $Q_{0}$ is a quadratic mapping satisfying (6) and (30). Then we have

$$
\begin{aligned}
\left\|Q(x)-Q_{0}(x)\right\|= & a^{-2 k}\left\|Q\left(a^{k} x\right)-Q_{0}\left(a^{k} x\right)\right\| \\
\leq & a^{-2 k}\left\|Q\left(a^{k} x\right)-g\left(a^{k} x\right)\right\| \\
& +a^{-2 k}\left\|Q_{0}\left(a^{k} x\right)-g\left(a^{k} x\right)\right\| \\
\leq & a^{-2 k} \sum_{n=0}^{\infty} a^{-2(n+1)} \phi_{0}\left(a^{n+k} x, 0\right) \\
= & a^{-2} \sum_{n=0}^{\infty} a^{-2(n+k)} \phi_{0}\left(a^{n+k} x, 0\right)
\end{aligned}
$$

for all $x \in X$ and for all positive integers $k$. Hence, letting $k \rightarrow \infty$ in the above inequality by (28) the tail part $\sum_{n=0}^{\infty} a^{-2(n+k)} \phi_{0}\left(a^{n+k} x, 0\right)=\sum_{n=k}^{\infty} a^{-2 n} \phi_{0}\left(a^{n} x, 0\right)$ goes to 0 . So we have

$$
Q(x)=Q_{0}(x)
$$

for all $x \in X$.

We remark that if $f(0)=0$ in Theorem 6, inequality (30) can be replaced by

$$
\|Q(x)-f(x)\| \leq \frac{1}{2} \sum_{n=0}^{\infty} a^{-2(n+1)} \phi\left(a^{n} x, 0\right) .
$$

Related with Theorem 6, we can also have the following theorem. And the proof is similar to that of Theorem 6 . 
Theorem 7. Let $\phi: X^{2} \rightarrow[0, \infty)$ be a function such that

$$
\sum_{n=0}^{\infty} a^{2 n} \phi\left(a^{-n} x, a^{-n} y\right)<\infty
$$

for all $x, y \in X$. Let $f: X \rightarrow Y$ be a mapping such that

$$
\begin{aligned}
& \| f(a x+b y)+f(a x-b y)-2 a^{2} f(x)-2 b^{2} f(y) \\
& +c[f(x+y)+f(x-y)-2 f(x)-2 f(y)] \| \\
& \quad \leq \phi(x, y)
\end{aligned}
$$

for a fixed rational number $a$ and fixed nonzero real numbers $b, c$ with $a \neq 1,0-1$ and $a^{2} \neq b^{2}$. Then there exists a unique quadratic mapping $Q: X \rightarrow Y$ such that

$$
\|Q(x)-f(x)-f(0)\| \leq \frac{1}{2} \sum_{n=0}^{\infty} a^{2(n+1)}\left[\phi\left(a^{-n} x, 0\right)+\phi(0,0)\right]
$$

for all $x \in X$.

For the stability problem of quadratic functional equations, we can show that many quadratic functional equations turn out to be types of (6) or to be deformed into the type of (6). For example, Gordji and Khodaei [13] investigated the following functional equation:

$$
\begin{aligned}
f(a x+b y)+f(a x-b y) & \\
= & \frac{b(a+b)}{2} f(x+y)+\frac{b(a+b)}{2} f(x-y) \\
& +\left(2 a^{2}-a b-b^{2}\right) f(x)+\left(b^{2}-a b\right) f(y), \\
f(a x & +b y)+f(a x-b y)=2 a^{2} f(x)+b^{2} f(y) .
\end{aligned}
$$

Indeed, the functional equation (44) can be written as

$$
\begin{aligned}
f(a x+b y)+f(a x-b y) & \\
& +c[f(x+y)+f(x-y)-2 f(x)-2 f(y)] \\
= & 2 a^{2} f(x)+2 b^{2} f(y),
\end{aligned}
$$

where $c=-b(a+b) / 2$. Hence the functional equations (44) and (45) are special cases of the functional equation (6).

As another example, Jun et al. [16] investigated the following functional equation:

$$
f(a x+y)+a f(x-y)=(a+1) f(y)+a(a+1) f(x),
$$

where $a$ is an integer with $a \neq-1,0$. Suppose that $f$ satisfies (47). Then clearly, $f$ is even, and hence the functional equation (47) can be deformed into

$$
\begin{aligned}
& f(a x+y)+f(a x-y) \\
& \quad+a[f(x+y)+f(x-y)-2 f(x)-2 f(y)] \\
& =2 a^{2} f(x)+2 f(y)
\end{aligned}
$$

for all $x, y \in X$. That is, we can transform (47) into the type of (6).

As an example of $\phi(x, y)$ in Theorems 6 and 7, we can take $\phi(x, y)=\epsilon\left(\|x\|^{p}\|y\|^{p}+\|x\|^{2 p}+\|y\|^{2 p}\right)$ which appeared in [17]. Then we can formulate the following corollary.

Corollary 8. Let $p$ be a real number with $p \neq 1$. Let $f: X \rightarrow$ $Y$ be a mapping such that

$$
\begin{aligned}
& \| f(a x+b y)+f(a x-b y)-2 a^{2} f(x) \\
& -2 b^{2} f(y)+c[f(x+y)+f(x-y)-2 f(x)-2 f(y)] \| \\
& \quad \leq \epsilon\left(\|x\|^{p}\|y\|^{p}+\|x\|^{2 p}+\|y\|^{2 p}\right)
\end{aligned}
$$

for a fixed rational number $a$ and fixed nonzero real numbers $b, c$ with $a \neq 1,0,-1$ and $a^{2} \neq b^{2}$. Then there exists a unique quadratic mapping $Q: X \rightarrow Y$ such that

$$
\begin{gathered}
\|Q(x)-f(x)-f(0)\| \leq \frac{\epsilon\|x\|^{2 p}}{2\left[1-a^{2(1-p)}\right]} \\
(p>1,|a|>1, \text { or } p<1,|a|<1), \\
\|Q(x)-f(x)-f(0)\| \leq \frac{\epsilon\|x\|^{2 p}}{2\left[1-a^{2(p-1)}\right]} \\
(p<1,|a|<1, \text { or } p>1,|a|>1)
\end{gathered}
$$

for all $x \in X$.

We remark that the functional equation (6) is not stable for $p=1$ in Corollary 8. The following example, which is a special case of the example in [18], shows that (6) is not stable for $p=1$ especially in the case of $a=2, b=1$, and $c=-1$. We give a proof for the reader's convenience.

Example 9. Let $t: \mathbb{R} \rightarrow \mathbb{R}$ be a mapping defined by

$$
t(x)= \begin{cases}x^{2}, & \text { if }|x|<1 \\ 1, & \text { otherwise }\end{cases}
$$

and define a mapping $f: \mathbb{R} \rightarrow \mathbb{R}$ by

$$
f(x)=\sum_{n=0}^{\infty} \frac{t\left(2^{n} x\right)}{4^{n}} .
$$

We will show that $f$ satisfies the functional inequality

$$
\begin{aligned}
& \| f(2 x+y)+f(2 x-y)-8 f(x)-2 f(y) \\
& -[f(x+y)+f(x-y)-2 f(x)-2 f(y)] \| \\
& \quad \leq \frac{640}{3}\left(|x||y|+|x|^{2}+|y|^{2}\right)
\end{aligned}
$$

for all $x, y \in \mathbb{R}$, but there do not exist a quadratic mapping $Q: \mathbb{R} \rightarrow \mathbb{R}$ and a positive constant $K$ such that

$$
\|Q(x)-f(x)\| \leq K\|x\|^{2}
$$

for all $x \in \mathbb{R}$. 
Note that $|f(x)| \leq 4 / 3$ for all $x \in \mathbb{R}$. For any mapping $g: \mathbb{R} \rightarrow \mathbb{R}$, let

$$
\begin{array}{r}
D g(x, y)=g(2 x+y)+g(2 x-y)-8 g(x) \\
-2 g(y)-[g(x+y)+g(x-y) \\
-2 g(x)-2 g(y)]
\end{array}
$$

for all $x, y \in \mathbb{R}$.

First, suppose that $1 / 16 \leq|x||y|+|x|^{2}+|y|^{2}$. Then $|D f(x, y)| \leq(640 / 3)\left(|x||y|+|x|^{2}+|y|^{2}\right)$ for all $x, y \in \mathbb{R}$.

Now suppose that $1 / 16>|x||y|+|x|^{2}+|y|^{2}$. Then there is a positive integer $m$ such that

$$
\frac{1}{2^{2 m+3}} \leq|x||y|+|x|^{2}+|y|^{2}<\frac{1}{2^{2 m+2}}
$$

and so

$$
2^{m}|x|<\frac{1}{2}, \quad 2^{m}|y|<\frac{1}{2}
$$

Hence, we have

$$
\begin{aligned}
& \left\{2^{m-1}(2 x \pm y), 2^{m-1}(x \pm y), 2^{m-1} x, 2^{m-1} y\right\} \\
& \quad \subseteq(-1,1) .
\end{aligned}
$$

Hence for any $n=0,1,2, \ldots, m-1$,

$$
D t\left(2^{n} x, 2^{n} y\right)=0 \text {, }
$$

and so

$$
\begin{aligned}
D f(x, y) & \leq \sum_{n=0}^{\infty} \frac{1}{4^{n}} \operatorname{Dt}\left(2^{n} x, 2^{n} y\right) \\
& =\sum_{n=m}^{\infty} \frac{1}{4^{n}} \operatorname{Dt}\left(2^{n} x, 2^{n} y\right) \\
& \leq \frac{40}{3 \times 2^{2 m}} \leq \frac{320}{3}\left(|x||y|+|x|^{2}+|y|^{2}\right) .
\end{aligned}
$$

Thus $f$ satisfies (53).

Suppose that there exist a quadratic mapping $Q: \mathbb{R} \rightarrow \mathbb{R}$ and a positive constant $K$ with (54). Since $|f(x)| \leq 4 / 3$,

$$
-K x^{2}-\frac{4}{3} \leq Q(x) \leq K x^{2}+\frac{4}{3}
$$

for all $x \in \mathbb{R}$, and since $Q$ is quadratic,

$$
-K x^{2}-\frac{4}{3 n^{2}} \leq Q(x) \leq K x^{2}+\frac{4}{3 n^{2}}
$$

for all $x \in \mathbb{R}$ and all natural numbers $n$. Hence, we have

$$
|Q(x)| \leq K x^{2}
$$

for all $x \in \mathbb{R}$, and so, by (54), we have

$$
|f(x)| \leq 2 K x^{2}
$$

for all $x \in \mathbb{R}$.
Take a positive integer $l$ such that $l>2 K$, and pick $x \in \mathbb{R}$ with $2^{l-1}|x|<1$. Then

$$
f(x)=\sum_{n=0}^{\infty} \frac{t\left(2^{n} x\right)}{4^{n}}>\sum_{n=0}^{l-1} \frac{t\left(2^{n} x\right)}{4^{n}}=\sum_{n=0}^{l-1} x^{2}=l x^{2}>2 K x^{2},
$$

which contradicts (64).

\section{Deforming Inequalities into the Type of (29)}

It turns out that lots of functional inequalities can be deformed into inequality (29). So we can regard inequality (29) as a typical form of a certain class of functional inequalities. In this point of view, we have a following systematic program to prove the generalized Hyers-Ulam stability of certain functional inequalities.

Step 1. Deform a given inequality into the type of (29) and get a modified bound function.

Step 2. Apply Theorem 6 for the modified bound function.

It should be remarked that if a functional inequality can be deformed into the type of (29), then a solution of the original functional equation is quadratic. And, it can be easily checked that the resulting unique quadratic mapping $Q$ in Step 2 also satisfies the original functional equation. So we don't need to worry anything about the given functional equation in our program. In this section, we illustrate just two of them.

First, we consider the following functional equation:

$$
\begin{gathered}
f(a x+y)+(a-1) f(x-y)-f(x+y) \\
=(a+2)(a-1) f(x)+(a-1) f(y)
\end{gathered}
$$

for some rational number $a$ with $a \neq 1,-1,0$.

Theorem 10. Let $\phi: X^{2} \rightarrow[0, \infty)$ be a function with (28). Let $f: X \rightarrow Y$ be a mapping satisfying $f(0)=0$ and

$$
\begin{aligned}
& \| f(a x+y)+(a-1) f(x-y)-f(x+y) \\
& -(a+2)(a-1) f(x)-(a-1) f(y) \| \leq \phi(x, y)
\end{aligned}
$$

for some rational number a with $a \neq 1,-1,0$. Then there exists a unique quadratic mapping $Q: X \rightarrow Y$ such that $Q$ satisfies (66) and

$$
\|Q(x)-f(x)\| \leq \sum_{n=0}^{\infty} a^{-2(n+1)}\left[\phi\left(a^{n} x, 0\right)+\frac{1}{2} \phi(0,0)\right]
$$

for all $x \in X$.

Proof. Setting $y=-y$ in (67), we have

$$
\begin{aligned}
& \| f(a x-y)+(a-1) f(x+y)-f(x-y) \\
& \quad-(a+2)(a-1) f(x)-(a-1) f(-y) \| \leq \phi(x,-y),
\end{aligned}
$$


and by (67) and (69), we have

$$
\begin{aligned}
& \| f(a x+y)+f(a x-y)-2 a^{2} f(x)-2 f(y) \\
& +(a-2)[f(x+y)+f(x-y)-2 f(x)-2 f(y)] \\
& +(a-1)[f(y)-f(-y)] \| \\
& \quad \leq \phi(x, y)+\phi(x,-y)
\end{aligned}
$$

for all $x, y \in X$. Letting $x=0$ in (67), we have

$$
\|(a-1)[f(y)-f(-y)]\| \leq \phi(0, y)
$$

for all $y \in X$. Hence, by (70) and (71), we have

$$
\begin{aligned}
& \| f(a x+y)+f(a x-y)-2 a^{2} f(x)-2 f(y) \\
& +(a-2)[f(x+y)+f(x-y)-2 f(x)-2 f(y)] \| \\
& \quad \leq \Phi(x, y)
\end{aligned}
$$

for all $x, y \in X$, where $\Phi(x, y)=\phi(x, y)+\phi(x,-y)+\phi(0, y)$. So by Theorem 6 , we get the result.

Remark 11. It would be interesting to see how Theorem 10 works well for a simple case of $\phi$. Take $\phi(x, y)=\epsilon>0$. Then the original inequality in Theorem 10 is

$$
\begin{gathered}
\| f(a x+y)+(a-1) f(x-y)-f(x+y) \\
-(a+2)(a-1) f(x)-(a-1) f(y) \| \leq \epsilon .
\end{gathered}
$$

After the deforming process, inequality (73) turns into the following new inequality which is standard in our sense:

$$
\begin{aligned}
& \| f(a x+y)+f(a x-y)-2 a^{2} f(x)-2 f(y) \\
& +(a-2)[f(x+y)+f(x-y)-2 f(x)-2 f(y)] \| \\
& \quad \leq 3 \epsilon .
\end{aligned}
$$

With $f(0)=0$, apply Theorem 6 or Theorem $7(\phi(x, y)=3 \epsilon$ in the theorems) to inequality (74); we get the following conclusion.

There exists a unique quadratic mapping $Q: X \rightarrow Y$ such that $Q$ satisfies (66) and

$$
\|Q(x)-f(x)\| \leq \frac{3 \epsilon}{2\left|a^{2}-1\right|} .
$$

Now, we consider the following functional equation:

$$
\begin{aligned}
& f(3 x+2 y)+2 f(3 x-2 y)-6 f(x-y) \\
& \quad=21 f(x)+6 f(y) .
\end{aligned}
$$

Theorem 12. Let $\phi: X^{2} \rightarrow[0, \infty)$ be a function with (28) for $a=3$. Let $f: X \rightarrow Y$ be a mapping satisfying $f(0)=0$ and

$$
\begin{aligned}
& \| f(3 x+2 y)+2 f(3 x-2 y)-6 f(x-y) \\
& -21 f(x)-6 f(y) \| \leq \phi(x, y) .
\end{aligned}
$$

Then there exists a unique quadratic mapping $Q: X \rightarrow Y$ such that $Q$ satisfies (76) and

$$
\|Q(x)-f(x)\| \leq \frac{1}{3} \sum_{n=0}^{\infty} 3^{-2(n+1)}\left[\phi\left(3^{n} x, 0\right)+6 \phi(0,0)\right]
$$

for all $x \in X$.

Proof. Setting $x=0$ in (77), we have

$$
\|f(2 y)+2 f(-2 y)-6[f(y)+f(-y)]\| \leq \phi(0, y)
$$

for all $y \in X$. Letting $y=-y$ in (79), we have

$$
\|2 f(2 y)+f(-2 y)-6[f(y)+6 f(-y)]\| \leq \phi(0,-y)
$$

for all $y \in X$. By (79) and (80), we have

$$
\|f(2 y)-f(-2 y)\| \leq \phi(0, y)+\phi(0,-y)
$$

for all $y \in X$. Hence by (81), we have

$$
\|f(y)-f(-y)\| \leq \phi\left(0, \frac{y}{2}\right)+\phi\left(0, \frac{-y}{2}\right)
$$

for all $y \in X$.

Letting $y=-y$ in (77), we get

$$
\begin{aligned}
& \| f(3 x-2 y)+2 f(3 x+2 y)-6 f(x+y) \\
& -21 f(x)-6 f(-y) \| \leq \phi(x,-y),
\end{aligned}
$$

and by (77) and (83), we have

$$
\begin{aligned}
& \| 3[f(3 x-2 y)+f(3 x+2 y)-18 f(x)-8 f(y)] \\
& -6[f(x+y)+f(x-y)-2 f(x)-2 f(y)] \\
& +6[f(y)-f(-y)] \| \\
& \quad \leq \phi(x, y)+\phi(x,-y)
\end{aligned}
$$

for all $x, y \in X$, and so by (82), we have

$$
\begin{aligned}
& \|[f(3 x-2 y)+f(3 x+2 y)-18 f(x)-8 f(y)] \\
& -2[f(x+y)+f(x-y)-2 f(x)-2 f(y)] \| \\
& \quad \leq \Phi(x, y)
\end{aligned}
$$

for all $x, y \in X$, where $\Phi(x, y)=(1 / 3)[\phi(x, y)+\phi(x,-y)]+$ $2[\phi(0, y / 2)+\phi(0,-y / 2)]$. So by Theorem 6 , we get the result.

\section{Conflict of Interests}

The authors declare that there is no conflict of interests regarding the publication of this paper.

\section{Acknowledgment}

The first author was supported by the research fund of Dankook University in 2013. 


\section{References}

[1] S. M. Ulam, A Collection of Mathematical Problems, Interscience Publisher, New York, NY, USA, 1964.

[2] D. H. Hyers, "On the stability of the linear functional equation," Proceedings of the National Academy of Sciences of the United States of America, vol. 27, pp. 222-224, 1941.

[3] T. Aoki, "On the stability of the linear transformation in Banach spaces," Journal of the Mathematical Society of Japan, vol. 2, no. 1-2, pp. 64-66, 1950.

[4] T. M. Rassias, "On the stability of the linear mapping in Banach spaces," Proceedings of the American Mathematical Society, vol. 72, no. 2, pp. 297-300, 1978.

[5] P. Găvruța, "A generalization of the Hyers-Ulam-Rassias stability of approximately additive mappings," Journal of Mathematical Analysis and Applications, vol. 184, no. 3, pp. 431-436, 1994.

[6] F. Skof, "Proprieta' locali e approssimazione di operatori," Rendiconti del Seminario Matematico e Fisico di Milano, vol. 53, no. 1, pp. 113-129, 1983.

[7] P. W. Cholewa, "Remarks on the stability of functional equations," Aequationes Mathematicae, vol. 27, no. 1, pp. 76-86, 1984.

[8] S. Czerwik, "On the stability of the quadratic mapping in normed spaces," Abhandlungen aus dem Mathematischen Seminar der Universität Hamburg, vol. 62, no. 1, pp. 59-64, 1992.

[9] C. G. Park, "On the stability of the quadratic mapping in Banach modules," Journal of Mathematical Analysis and Applications, vol. 276, no. 1, pp. 135-144, 2002.

[10] G. Eskandani, H. Vaezi, and Y. N. Dehghan, "Stability of a mixed additive and quadratic functional equation in non-archimedean banach modules," Taiwanese Journal of Mathematics, vol. 14, no. 4, pp. 1309-1324, 2010.

[11] F. Moradlou, H. V. Vaezi, and G. Z. Eskandani, "Hyers-UlamRassias stability of a quadratic and additive functional equation in quasi-Banach spaces," Mediterranean Journal of Mathematics, vol. 6, no. 2, pp. 233-248, 2009.

[12] J. M. Rassias, "Solution of the Ulam stability problem for Euler-Lagrange quadratic mappings," Journal of Mathematical Analysis and Applications, vol. 220, no. 2, pp. 613-639, 1998.

[13] M. E. Gordji and H. Khodaei, "On the generalized hyers-ulamrassias stability of quadratic functional equations," Abstract and Applied Analysis, vol. 2009, Article ID 923476, 11 pages, 2009.

[14] M. E. Gordji, M. B. Savadkouhi, and C. Park, "Quadratic-quartic functional equations in RN-spaces," Journal of Inequalities and Applications, vol. 2009, Article ID 868423, 14 pages, 2009.

[15] K. W. Jun, H. M. Kim, and I. S. Chang, "On the Hyers-Ulam stability of an Euler-Lagrange type cubic functional equation," Journal of Computational Analysis and Applications, vol. 7, no. 1, pp. 21-33, 2005.

[16] K. W. Jun, H. M. Kim, and J. Son, "Generalized Hyers-Ulam stability of a quadratic functional equation," Functional Equations in Mathematical Analysis, vol. 2012, pp. 153-164, 2012.

[17] K. Ravi, M. Arunkumar, and J. M. Rassias, "Ulam stability for the orthogonally general Euler-Lagrange type functional equation," International Journal of Mathematics and Statistics, vol. 3, no. 8, pp. 36-46, 2008.

[18] H. M. Kim, J. M. Rassias, and J. Lee, "Fuzzy approximation of Euler-Lagrange quadratic mappings," Journal of Inequalities and Applications, vol. 2013, article 358, 15 pages, 2013. 




Advances in

Operations Research

mansans



The Scientific World Journal
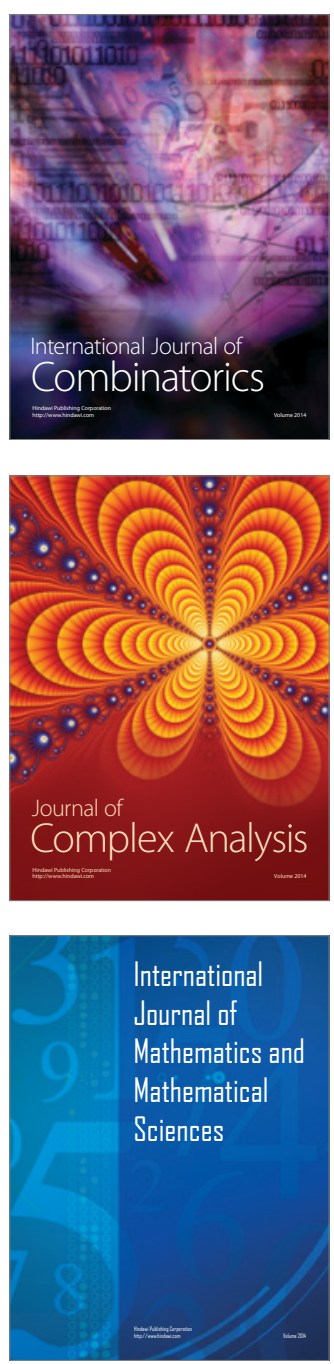
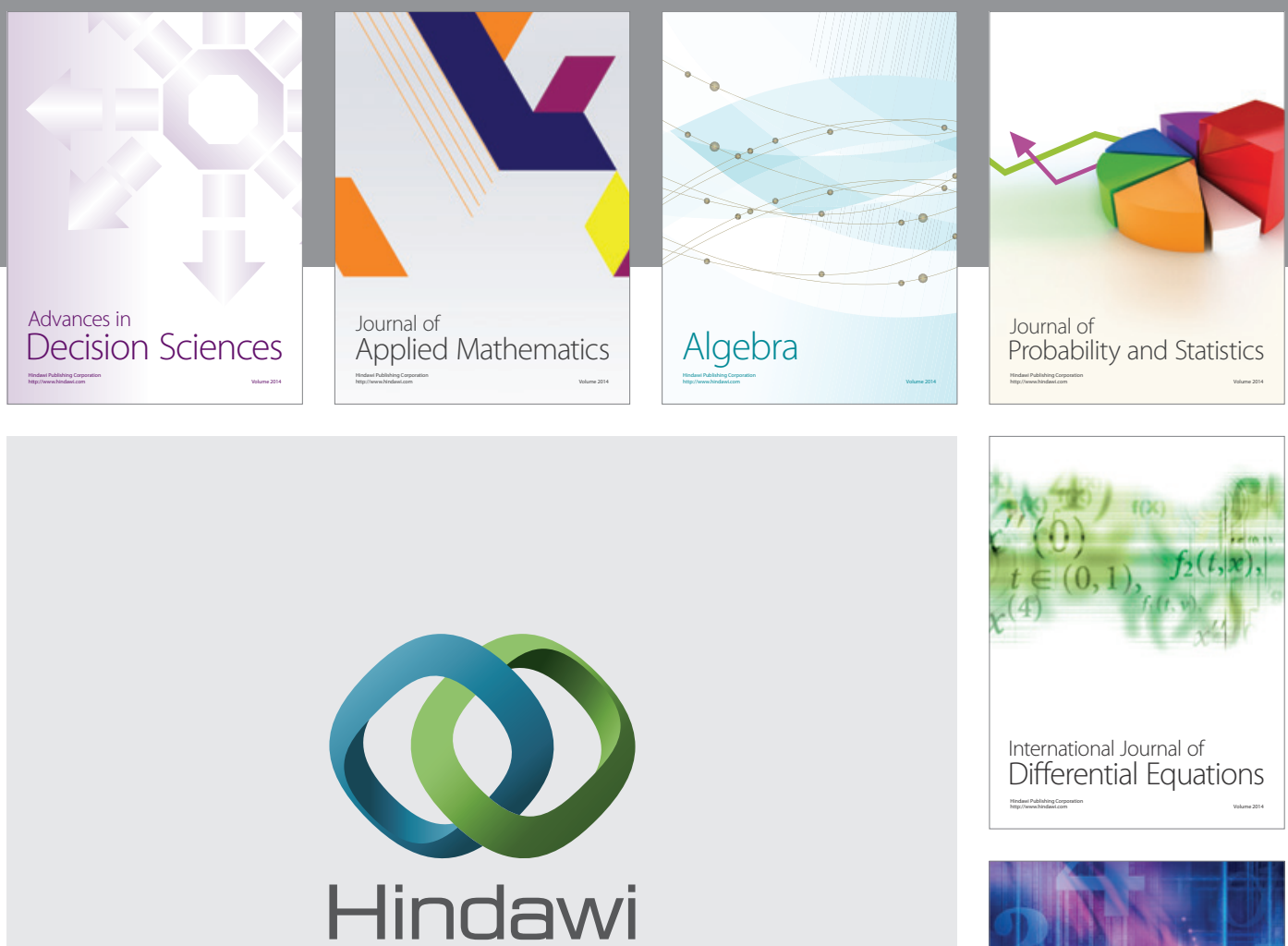

Submit your manuscripts at http://www.hindawi.com
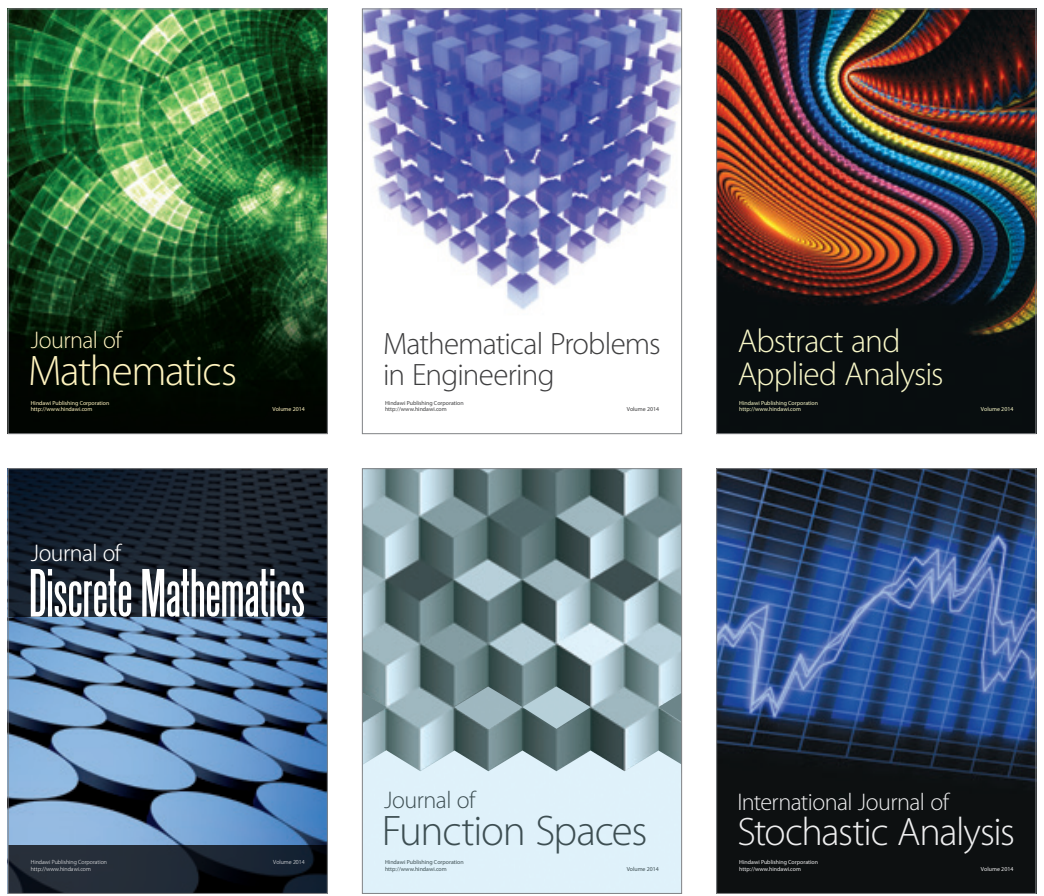

Journal of

Function Spaces

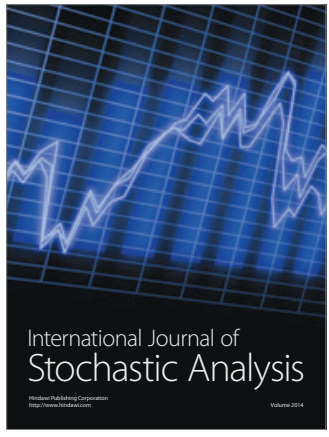

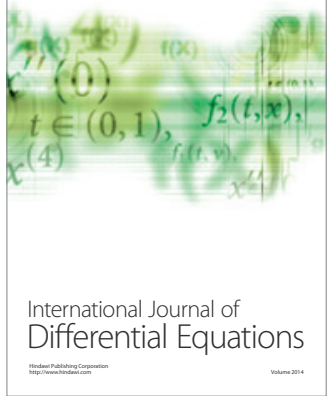
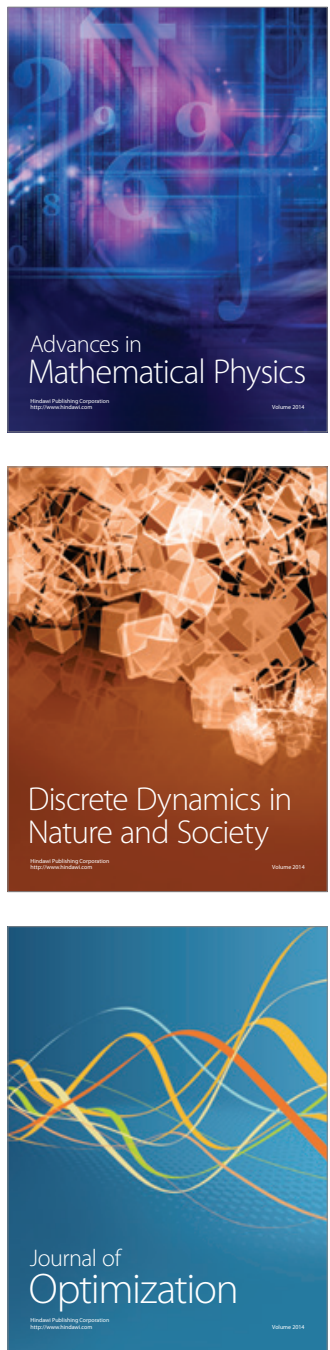